\title{
Packet signaling for systematic coding schemes with arbitrary data-to-repair-packet connection setups
}

\author{
César Díaz, Julián Cabrera, Fernando Jaureguizar, and Narciso García
}

\begin{abstract}
We consider the introduction of three different methods to signalize which data packets are used to generate each repair packet in systematic coding schemes employing flexible connection setups: a first one based on a mask, a second one on explicitly pointing out the links between the two packet streams, and a third one on sharing the signaling load between both flows. These methods have been evaluated in terms of the bandwidth required to put them into practice. The evaluation results show that the explicit method introduces less bandwidth, whereas the one sharing the load handles it more smartly.
\end{abstract}

Keywords-AL-FEC, packet signaling, bandwidth

\section{INTRODUCTION}

In transmission systems over IP networks where systematic Application-Layer Forward Error Correction (AL-FEC) codes are introduced to deal with channel impairments, one of the main design concerns is the amount of extra bandwidth devoted to the repair flow [1]. One key goal is then to avoid expending too much bandwidth on signalizing which data packets are protected by which repair packets. To that end, RFCs and other standardization documents specify, both within and outside the FEC framework defined in the RFC 6363 [2], compact, efficient methods to better accommodate this information, regarding the particularities of each code. For instance, the SMPTE 2022 standard [3] resorts to the dimensions of the matrix typical of the Pro-MPEG COP3 codes to signalize the links between the two flows. Or the RFC 6682 [4], which incorporates all the required parameters for the receiver to replicate the Raptor/Raptor Q encoding scheme used to create the redundant flow, so that the packets involved are properly arranged and the recovery process can be carried out.

Nevertheless, these signaling mechanisms are only possible if the connections between repair and data packets respond to nonflexible, replicable connection setups. This is not the case, for instance, when the links are set randomly, or when they correspond with exterior criteria, e.g., they are the result of packet-level distortion minimization problems within unequal error protection strategies [5]

In this paper, we evaluate three different methods of signalizing arbitrary repair-to-data-packet connections in terms of the bandwidth required to put them into practice.

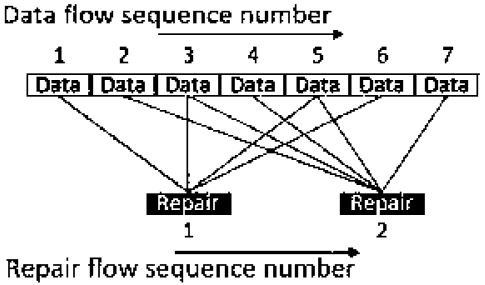

Fig. 1 Example of connections between data and repair packets

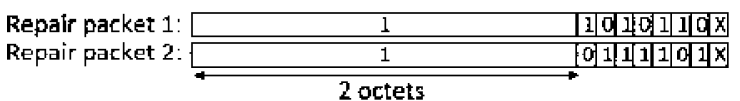

Fig.2 Mask signaling method

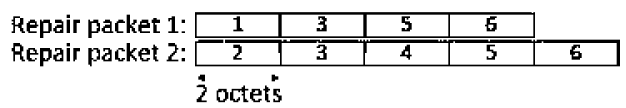

Fig.3 Explicit signaling method

\section{Signaling METHODS}

In this section, we describe the three signaling methods, and include a calculation of the bandwidth that each of them requires. Moreover, we resort to the example depicted in Fig.1 to better show how they work.

\section{A. Mask signaling method}

This is the only standardized procedure to signalize flexible connection setups, however only for protection blocks of up to 48 data packets [6]. What is described here represents an extension to this method. Each FEC packet carries the following signaling information to identify the data packets that it protects: (i) the minimum sequence number among the $k$ data packets in the protection block, $\mathrm{SN}_{\text {base }}(2$ octets); and (ii) a mask made up of $k$ bits. Setting the $p^{\text {th }}$ bit means that the repair packet was generated using the $\left(\mathrm{SN}_{\text {base }}+p-1\right)^{\text {th }}$ data packet in the protection block $([k / 8\rceil$ octets, as the number of octets has to be integral). Fig. 2 shows this method for the example in Fig. 1.

\section{B. Explicit signaling method}

Each repair packet transports: (i) the sequence number of all the data packets that it protects ( 2 octets per data packets protected). Fig. 3 shows this method for the example in Fig. 1. 


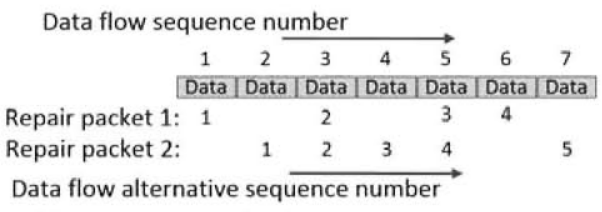

Fig.4 Alternative sequence numbering

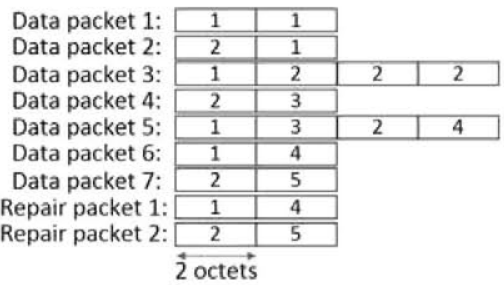

Fig.5 Shared-load signaling method

\section{Shared-load signaling method}

Firstly, this method generates an alternative, independent sequence numbering of the data packets protected by each FEC packet. To that end, the packet with the minimum real sequence number among the ones protected by a given repair packet receives an alternative base sequence number, which is incremented by one for the data packet in this same set with the second minimum real sequence number, and so on. This is depicted in Fig.4. This procedure allows the consideration of the data packets in the same set as if they laid consecutively in the stream, enabling a compact signaling in the sense of [3].

Contrary to other methods, the signaling load needs to be shared between the FEC packets and the data packets. This can be easily carried out through a header extension that in no way compromises the systematic nature of the code, as the original content is not modified. Fig. 5 shows this method for the example in Fig.1.

Repair packets carry the following signaling information: (i) the minimum sequence number among the packets that it protects (2 octets); and (ii) the number of data packets protected ( 2 octets).

Data packets include the following signaling information: (i) the sequence number of the repair packets that were created for its protection ( 2 octets per FEC packet); and (ii) the alternative sequence number derived from the new numbering ( 2 octets per FEC packet).

\section{EVALUATION OF THE SIGNALING METHODS AND CONCLUSIONS}

In this section, we compute the bandwidth associated to the use of each signaling method. To that end, we consider three possible scenarios, in which we assume that all data packets are connected to one, two or three repair packets.

Fig.6 shows the average bandwidth required per packet (data and repair) for packet signaling, regarding the number of data packets in the protection block. An overall extra bandwidth for protection purposes of $15 \%$ of the bitrate of the main flow is assumed.

We can notice that the mask signaling method performance does not depend on the number of repair packets used to

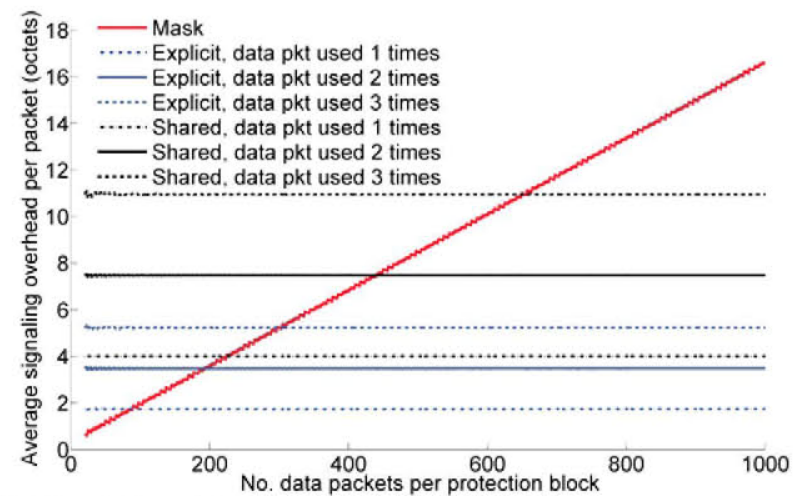

Fig.6 Evolution of the average bandwidth per packet

protect each data packet. In addition, with this procedure, the signaling load per packet grows quasi-linearly with the number of data packets in the protection block. If this number is kept low, this method is very well suited. However, it becomes less efficient as the number of data packets increases. On the contrary, the performance of the other two methods remains rather constant with the size of the protection block.

We also see that for a sufficiently high number of data packets, the explicit method requires the lowest overall signaling bandwidth. However, this method might not handle it conveniently. The load of a single FEC packet directly depends on the number of data packets protected by it, which might differ enormously from repair packet to repair packet. Thus, although it is averagely efficient, this behavior may lead to undesirable events, like exceeding the network's MTU.

Regarding the shared-load signaling method, we observe that it demands more resources than the latter. Nevertheless, it distributes the associated load more efficiently among packets. Thus, the amount of extra information added to each packet is very low and fairly constant.

Finally, we can conclude that, as already said, the method based on the mask is perfectly appropriate for small protection blocks. As this block size increases, the explicit signaling method becomes the most suitable, in terms of the extra bandwidth required. However, particular scenario features might require a more smartly handled packet signaling, making the shared-load signaling method the best choice.

\section{REFERENCES}

[1] F. Battisti, M. Carli, E. Mammi, and A. Neri, "A study on the impact of AL-FEC techniques on TV over IP Quality of Experience," EURASIP Journal on Advances in Signal Processing, no. 1, pp. 86, 2011.

[2] M. Watson, A. Begen, and V. Roca, "Forward Error Correction (FEC) Framework," RFC 6363, Oct. 2011

[3] SMPTE ST 2022-1, "Forward error correction for real-time video/audio transport over IP networks," May 2007.

[4] M. Watson, T. Stockhammer, and M. Luby, "RTP Payload Format for Raptor Forward Error Correction (FEC)," RFC 6682, Aug. 2012.

[5] C. Diaz, J. Cabrera, F. Jaureguizar, and N. Garcia, "Adaptive protection scheme for MVC-encoded stereoscopic video streaming in IP-based networks," in 2012 IEEE Visual Communications and Image Processing (VCIP), Nov. 2012, pp. 1-6.

[6] A. Li, "RTP Payload Format for Generic Forward Error Correction," RFC 5109, Dec. 2007. 